\title{
Steuergesetzrevision 2008 im Kanton Thurgau
}

\author{
Mit der neuesten Gesetzesrevision setzt der Kanton Thurgau wesentliche \\ Punkte der Unternehmenssteuerreform II des Bundes, die am 24. Februar 2008 \\ vom Volk angenommen wurde, bereits ab 2008 auf kantonaler Ebene um.
}

Anhand einiger Beispiele werden die Auswirkungen dieser Steuergesetzrevision auf Ärzte und Arztpraxen aufgezeigt.

Adrian Hartmann

Korrespondenz:

Adrian Hartmann, dipl. Treuhandexperte

FMH Treuhand Services

Marktplatz 6

$\mathrm{CH}-8570$ Weinfelden
Seit einigen Jahren setzt der Kanton Thurgau seine Ende der 90er Jahre entwickelte und beschlossene Steuerstrategie um. Hauptziele dieser Strategie sind eine tendenziell sinkende Steuerbelastung, attraktive steuerliche Rahmenbedingungen für die Wirtschaft und ein modernes und attraktives Steuerrecht sowohl für natürliche als auch für juristische Personen.

Die Umsetzung dieser Steuerstrategie erfolgt laufend über mehrere Jahre hinweg. Eine unauffällige Politik der kleinen Schritte ohne den grossen Wurf wie z. B. im Kanton Obwalden und daher abseits des publizistischen Interesses. Trotzdem hat der Thurgau einige interessante Neuerungen und Erleichterungen eingeführt und in gewissen Punkten die sogenannte «Unternehmenssteuerreform II» des Bundes bereits vorweggenommen und umgesetzt.

\section{Halbsteuersatzverfahren für Beteiligungserträge}

Zur Milderung der wirtschaftlichen Doppelbelastung werden ab der Steuerperiode 2007 ausgeschüttete und versteuerte Gewinne aus Kapitalgesellschaften und Genossenschaften mit Sitz in der Schweiz zum halben Satz des steuerbaren Gesamteinkommens besteuert, sofern der Steuerpflichtige mit mindestens 5\% (Bund 10\%) am Aktien-, Grund- oder Stammkapital beteiligt ist.

Ein Berechnungsbeispiel soll verdeutlichen, was konkret mit dem sogenannten «Halbsteuersatzverfahren» gemeint ist: Eine verheiratete, im Kanton Thurgau steuerpflichtige Person ist zu 20\% an einer Aktiengesellschaft mit Sitz in der Schweiz (z.B. Kanton Zürich) beteiligt. Sie erhält aus dieser Beteiligung eine Dividende von Fr. 30000. Ihr gesamtes steuerbares Einkommen (Lohn, Zinsen, Dividende, Eigenmietwert abzüglich Schuldzinsen, Abzüge usw.) beträgt Fr. 157000.

Der Progressionssatz für ein steuerbares Einkommen von Fr. 157000 beträgt 5,7918\% (für gemeinsam steuerpflichtige Ehegatten). Die Steuerberechnung lautet somit:

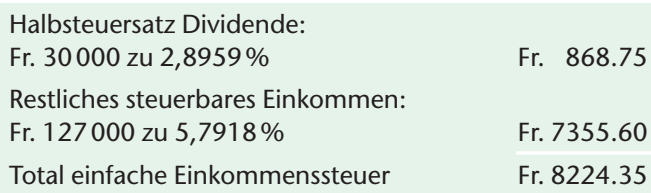

Die Steuerersparnis beträgt somit Fr. 868.75 einfache Steuer. Multipliziert mit dem Steuerfuss der Wohnsitzgemeinde (z. B. Frauenfeld: 298\%), ergibt sich eine totale Steuerersparnis von Fr. $2588.90(2,98 \times$ Fr. 868.75) .

\section{Besteuerung von Liquidationsgewinnen}

Liquidationsgewinne bei Aufgabe der selbständigen Erwerbstätigkeit werden ab 2008 (Bund ab 2009) privilegiert besteuert. Erfolgt die Aufgabe der selbständigen Erwerbstätigkeit nach dem vollendeten 55. Altersjahr oder infolge Invalidität, so werden die in den letzten zwei Geschäftsjahren realisierten stillen Reserven separat $\mathrm{zu}$ einem Fünftel des ordentlichen Tarifs, mindestens aber zu $3 \%$ (Bund 2\%) besteuert. Dies bringt beim Verkauf oder bei der Aufgabe einer Praxis erhebliche Vorteile.

Ein Beispiel soll dies verdeutlichen: Dr. Franz Muster, verheiratet, verkauft im Alter von 63 Jahren seine Arztpraxis an einen Nachfolger. Der Kaufpreis umfasst u.a. folgende Positionen:

$\begin{array}{ll}\text { Medikamente, Verbandsmaterial usw. } & \text { Fr. } 35000 \\ \begin{array}{l}\text { Praxiseinrichtungen, Geräte, } \\ \text { Instrumente usw. }\end{array} & \text { Fr. } 80000 \\ \text { Goodwill } & \text { Fr. } 65000 \\ \text { Total } & \text { Fr. } 180000\end{array}$

Der Buchwert für Medikamente, Praxiseinrichtungen usw. in der letzten Bilanz von Dr. Franz Muster beträgt Fr. 40000. Beim Verkauf werden somit stille Reserven im Umfang von Fr. 140000 realisiert. Das übrige steuerbare Einkommen von Dr. Muster im Jahre des Praxisverkaufes beträgt Fr. 160000. 


\begin{tabular}{|c|c|c|}
\hline Besteuerung alt & & \\
\hline $\begin{array}{l}\text { Auflösung / } \\
\text { Realisation stiller Reserven }\end{array}$ & Fr. 140000 & \\
\hline.$/$ AHV-Beiträge $(9,5 \%)$ & Fr. $\quad 13300$ & \\
\hline $\begin{array}{l}\text { Steuerbarer } \\
\text { Liquidationsgewinn }\end{array}$ & Fr. 126700 & \\
\hline $\begin{array}{l}\text { Restliches } \\
\text { steuerbares Einkommen }\end{array}$ & Fr. 160000 & \\
\hline $\begin{array}{l}\text { Steuerbares Einkommen } \\
\text { total }\end{array}$ & Fr. 286700 & \\
\hline $\begin{array}{l}\text { zum Satz von } 6,91 \% \\
=\text { Einfache Steuer }\end{array}$ & & Fr. 19811 \\
\hline Besteuerung neu & & \\
\hline $\begin{array}{l}\text { Steuerbarer } \\
\text { Liquidationsgewinn } \\
\text { (wie oben) }\end{array}$ & Fr. 126700 & \\
\hline $\begin{array}{l}\text { zum Satz von } 3 \% \\
=\text { Einfache Steuer }\end{array}$ & & Fr. $\quad 3801$ \\
\hline $\begin{array}{l}\text { Restliches } \\
\text { steuerbares Einkommen }\end{array}$ & Fr. 160000 & \\
\hline $\begin{array}{l}\text { zum Satz von } 5,83 \% \\
=\text { Einfache Steuer }\end{array}$ & & Fr. 9328 \\
\hline Total einfache Steuer neu & & Fr. 13129 \\
\hline
\end{tabular}

\section{Besteuerung von Kapitalleistungen aus Vorsorge}

Bis Ende 2007 erfolgte die Besteuerung von Kapitalleistungen aus Vorsorge zum Satz von 1/17 der Kapitalleistungen, mindestens aber $\mathrm{zu} 2 \%$ für Verheiratete und zu 2,5\% für Alleinstehende. Bei hohen Beträgen konnte dies zu einem Steuersatz von über 7\% (einfache Steuer) bzw. zu einer Gesamtsteuerbelastung von über $21 \%$ der erhaltenen Leistung führen.

Neu führt der Kanton Thurgau ab 2008 eine «Flat Rate Tax» für Kapitalleistungen aus Vorsorge ein. Unabhängig von der Höhe der Kapitalleistung beträgt der Steuersatz neu einheitlich $2 \%$ für Verheiratete und 2,4\% für Alleinstehende.

Auch zu diesem Punkt ein Berechnungsbeispiel zur Verdeutlichung: Dr. Max Beispiel, ledig, wohnhaft in Frauenfeld, lässt sich mit 64 Jahren einen Teil seines BVG-Alterskapitals in Form einer Kapitalleistung ausbezahlen.
Auszahlungsbetrag: Fr. 750000

Besteuerung alt

Satzbestimmung: Fr. 750000 : $17=$ Fr. $44118=4,45 \%$

Steuerberechnung:

Fr. 750000 zu $4,45 \%=$

Fr. 33375

Total Steuerbelastung

(Frauenfeld 298\%)

Fr. 99458

Besteuerung neu

Steuerberechnung:

Fr. 750000 zu 2,4\%

Total Steuerbelastung

(Frauenfeld 298\%)

Fr. 18000

Fr. 53640

Dieser Absatz gilt nur für den Kanton Thurgau und nicht für die Direkte Bundessteuer!

\section{Fazit}

Mit den vorgängig angeführten Neuerungen hat der Kanton Thurgau in verschiedenen Teilbereichen die Besteuerung einerseits vereinfacht und andererseits ganz erheblich gesenkt. Dies ist ein wichtiger Schritt in die richtige Richtung, insbesondere, was die Milderung der Doppelbelastung von Dividendenerträgen anbelangt (Besteuerung erst als Gewinn in der Gesellschaft und anschliessend als Einkommen beim Aktionär).

Ganz allgemein kann aber auch gesagt werden, dass unser Kanton aufgrund der verschiedenen Revisionen des Steuergesetzes heute ein aus steuerlicher Sicht attraktiver Standort geworden ist. Dieser Umstand zeigt sich auch deutlich anhand des gesamtschweizerischen Steuerindexes. Dort rangiert der Kanton Thurgau im Jahre 2006 auf dem beachtlichen 5. Platz. Noch im Jahre 2001 belegte er lediglich Platz 14.

Es bleibt zu hoffen, dass die Verantwortlichen an ihrer bisherigen Strategie festhalten und weitere Schritte zur Entlastung der Steuerzahler in die Wege leiten. Insbesondere sei hier die Senkung der Steuerfüsse (Kanton, Gemeinden usw.) erwähnt. 